\title{
Psoriasis, Anti-Tumor Necrosis Factor Therapy, and Tuberculosis: Report of Three Challenging Cases and Literature Review
}

Caius Solovan • Elena Chiticariu

To view enhanced content go to www.infectiousdiseases-open.com Received: November 26, 2012 / Published online: February 15, 2013

(C) The Author(s) 2013. This article is published with open access at Springerlink.com

\section{ABSTRACT}

Introduction: The era of biologic therapies has provided new options for the treatment of chronic plaque psoriasis. However, safety concerns have led to intensive screening and monitoring of patients receiving anti-tumor necrosis factor alpha (anti-TNF-alpha) agents.

Methods: The authors describe the cases of three patients with moderate to severe psoriasis treated with anti-TNF agents, with challenging diagnostic and treatment aspects regarding tuberculosis (TB) infection, a serious adverse event associated with this type of treatment. The cases are discussed in the context of a comprehensive literature review describing the risk of TB associated with the use of TNF inhibitors. A critical review of the

C. Solovan · E. Chiticariu $(\varangle)$

Department of Dermatology, University of Medicine and Pharmacy "Victor Babes" Timisoara, Marasesti 5, 300077 Timisoara, Romania

e-mail: elena.chiticariu@yahoo.com

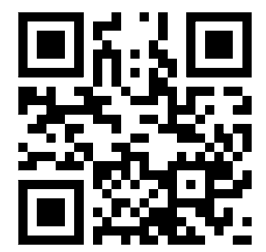

Enhanced content for this article is

available on the journal web site:

www.infectiousdiseases-open.com clinical trials that have tested the safety of these agents is also presented.

Results: One patient, who tested negatively for latent TB infection (LTBI) during screening, developed active TB under adalimumab therapy. For two other patients the diagnosis and management of LTBI in relation to antiTNF therapy represented a challenge. Although clinical trials involving the use of anti-TNF therapy for psoriasis haven't demonstrated a high TB incidence, active TB is continuously reported in association with this treatment.

Conclusions: Findings from clinical practice and the scientific literature indicate that antiTNF therapies are associated with an increased risk of $\mathrm{TB}$, and close monitoring of patients is needed.

Keywords: Adalimumab; Biologic therapy; Dermatology; Etanercept; Infliximab; Psoriasis; Tuberculosis; Tumor necrosis factor

\section{INTRODUCTION}

Psoriasis is a chronic inflammatory systemic disease predominantly affecting the skin and joints. The prevalence ranges between $0.9 \%$ 
(United States) and 8.5\% (Norway) [1]. Skin lesions are the major manifestation of the disease. They are described as scaling and erythematous plaques that may be pruritic or painful and cause significant quality of life issues [2].

The new era of biologic therapies offers outstanding options for the treatment of chronic plaque psoriasis, and these agents have proved to be remarkable in improving patient quality of life compared with classical antipsoriatic treatments. However, despite the high efficacy, there have always been concerns regarding the safety of these agents as all antitumor necrosis factor alpha (anti-TNF-alpha) agents have been associated with activation of latent tuberculosis infection (LTBI) in a relatively short period of time [3]. According to World Health Organization (WHO), the global incidence of tuberculosis (TB) is estimated to 125 cases per 100,000 population [4]. The progression or reactivation of $\mathrm{TB}$ should be expected and such concerns have led to intensive screening and monitoring of patients receiving anti-TNF therapies [5]. Current screening includes medical history, chest X-ray, and tests for evaluating the immunologic response to the presence of Mycobacterium tuberculosis, such as the tuberculin skin test (TST) and interferon gamma release assays (IGRAs) [6]. Current guidelines recommend TST as the main screening tool for LTBI in patients with psoriasis before initiation of anti-TNF therapy, but there is a lack of consensus on the interpretation of TST in this group of patients [7-9]. The European S3 guidelines recommend the use of either TST or IGRAs or both for LTBI detection [10]. However, as TST may produce false-positive results, the newest recommendations suggest the use of IGRAs [11].
Despite the screening programs for LTBI identification prior to anti-TNF therapy, the risk of developing active TB is still present. We report a patient with psoriasis who had a negative TST during screening and later developed active TB under adalimumab therapy. We also report two patients with challenging aspects regarding the diagnosis and management of LTBI in relation to antiTNF therapy. Additional evidence from a review of the literature is also discussed.

\section{CASE STUDIES}

Patient characteristics, TB status, and treatment received for all three case studies are summarized in Table 1.

\section{Case 1}

A 57-year-old man presented with a 18-year history of severe chronic plaque psoriasis. The patient was hypertensive. He was previously treated with systemic methotrexate and topical antipsoriatic therapies. He did not report any known contact with a case of active TB.

Due to the poor response to classical treatments for psoriasis, adalimumab was recommended according to current guidelines [2]. All screening tests were within normal ranges, including a negative TST (3 mm induration) and chest X-ray. Therefore, adalimumab therapy was initiated without antituberculous chemoprophylaxis. The patient showed a good and stable response; the Psoriasis Area and Severity Index (PASI) decreased from 36 to 9 in 12 weeks, and all lesions were cleared after 6 months of treatment.

After 18 months of biologic therapy, the patient complained of a mild but persistent 


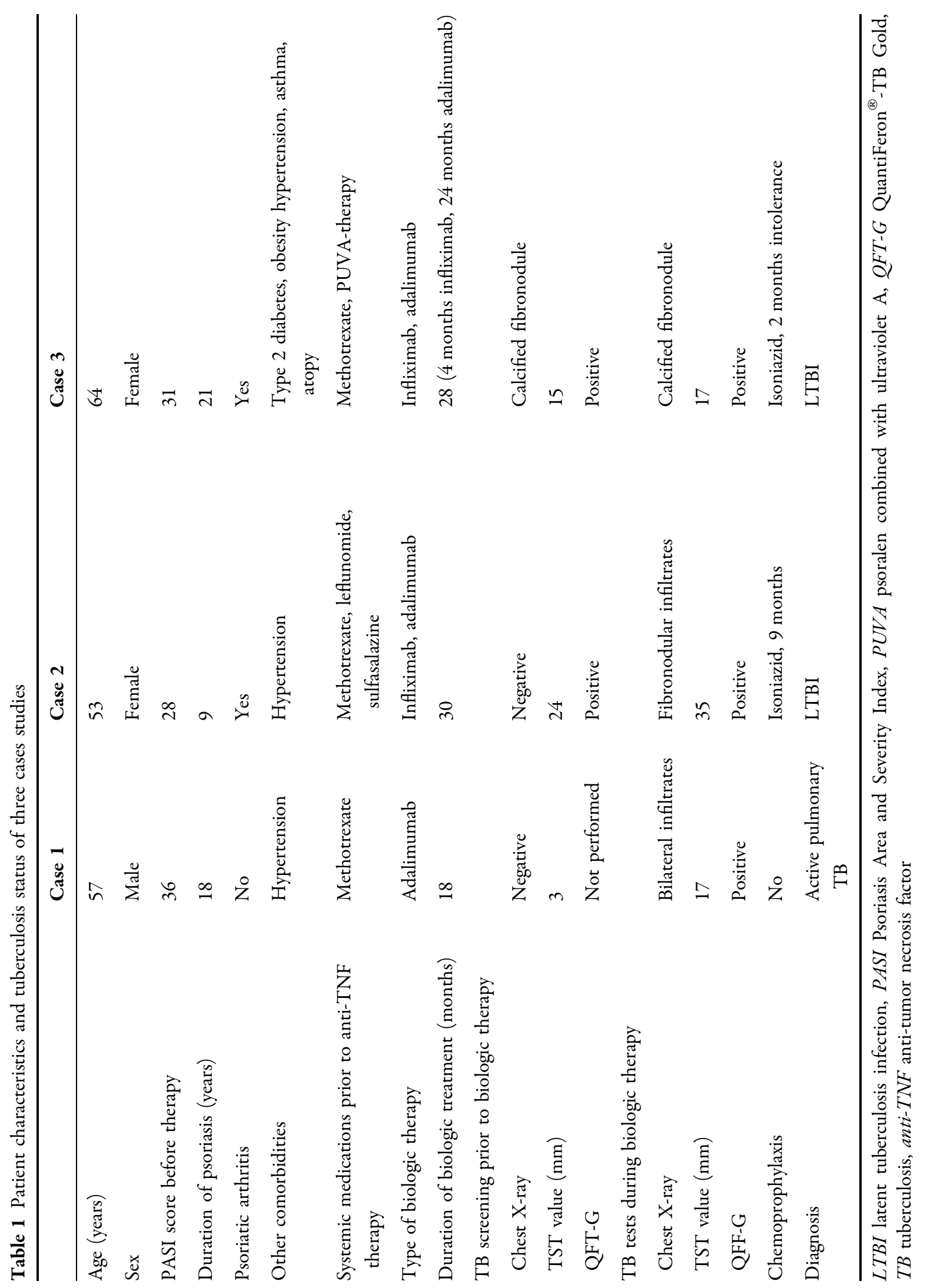


cough and loss of appetite. A subsequent TST was positive $(17 \mathrm{~mm})$. QuantiFeron ${ }^{\circledR}$-TB Gold (QFT-G) test (Cellestis Inc., Valencia, CA, USA) was also positive. Chest X-ray and computed tomography (CT) both showed bilateral pulmonary infiltrates. Routine laboratory examinations, including complete blood count and biochemical profile, were within normal limits. The patient was referred to a pulmonologist who confirmed active pulmonary TB with positive microbiology. The patient discontinued adalimumab and tuberculostatic treatment was prescribed. After a 6-month course of a multidrug anti-TB regimen, the pulmonary lesions were completely cleared but the psoriasis progressively worsened. With the patient's consent and the pneumologist's approval, adalimumab was resumed with close follow-up.

After 6 months of follow-up, there was a marked improvement in the patient's psoriasis and no report of any other side effects. Close monitoring of the patient will continue in order to rule out $\mathrm{TB}$ recurrence.

\section{Case 2}

A 53-year-old woman presented with a 9-year history of psoriasis vulgaris and psoriatic arthritis. She was previously treated with systemic methotrexate, leflunomide, sulfasalazine, and topical antipsoriatic therapies. She did not report any contact with a case of active TB.

The patient was screened before administration of biologic therapy. The patient's TST value was $24 \mathrm{~mm}$. Chest X-ray was negative. Clinical examination and routine laboratory tests were normal. Chemoprophylaxis with isoniazid (300 mg/ day, 9 months) was prescribed, which was initiated 1 month before anti-TNF therapy.
Subsequent treatment with infliximab was associated with a good response and complete clearing of skin lesions. Annual TST testing remained high in two repeated determinations (25, respectively $30 \mathrm{~mm}$ ). No side effects were noted in the first 2 years of treatment.

After 30 months of biologic therapy, the TST was $35 \mathrm{~mm}$, QFT-G was also positive, and a chest $\mathrm{X}$-ray showed two pulmonary nodular lesions. CT showed two fibronodular infiltrates in the inferior lobe of left lung and middle lobe of the right lung. Routine laboratory tests were within normal limits. The patient was asymptomatic, but was referred to a pneumologist who, based on clinical suspicion, recommended interruption of anti-TNF therapy and initiation of a tuberculostatic regimen. However, the sputum specimens were negative for $M$. tuberculosis by smear and culture, and active TB was finally infirmed. The patient was diagnosed with LTBI, resuming biologic therapy with another biologic agent: etanercept. The patient developed a persistent injection-site reaction after four doses of etanercept, a side effect that led to cessation of this anti-TNF treatment and initiation of adalimumab as an alternative treatment. The patient's condition is currently stable, with a continued response to adalimumab and no side effects after 6 months of follow-up. Close monitoring will continue in order to rule out reactivation of LTBI.

\section{Case 3}

A 64-year-old woman presented with a 21-year history of psoriasis. She suffered from psoriatic arthritis, type 2 diabetes mellitus, asthma, hypertension, atopy, and obesity. The patient reported allergic reactions to various medications, including penicillin, mometasone furoate, and aspirin. She had previously received systemic methotrexate and psoralen 
combined with ultraviolet A (PUVA) therapy and did not report any known contact with a case of active TB.

Prior to initiation of anti-TNF treatment with infliximab, she had undergone a TST with an induration of $15 \mathrm{~mm}$ and a positive QFT-G assay. Chest X-ray showed a calcified left apical fibronodule. Physical examination did not reveal any pathological findings. Routine laboratory tests were within normal range. The patient was diagnosed with LTBI and chemoprophylaxis with isoniazid $300 \mathrm{mg} /$ day was prescribed. After 2 months of isoniazid, she developed erythema multiforme and treatment was stopped. An attempt was made to reintroduce the chemoprophylactic treatment but the skin lesions reappeared.

Due to the severity of her condition (severe psoriasis with a PASI score of 31 and psoriatic arthritis), she continued infliximab therapy with close pneumology follow-up. After the fourth infusion, she developed an anaphylaxislike reaction to infliximab. The drug was discontinued and the patient was switched to adalimumab. The patient was treated successfully with adalimumab for 2 years without side effects. Monitoring will continue in order to rule out active TB.

\section{DISCUSSION}

The advent of anti-TNF agents has revolutionized the therapeutic approach to psoriasis and other inflammatory disorders. However, as these therapies have become widely used in clinical practice, $\mathrm{TB}$ is increasingly recorded. The authors presented three cases of patients with challenging aspects regarding the risk of $\mathrm{TB}$ related to anti-TNF therapy. The first patient, excluding his psoriasis, was an otherwise healthy individual with no predisposing factors for TB. A TST response of $3 \mathrm{~mm}$ during the screening was considered negative. This suggests that even healthy individuals with no predisposing factors or evidence of LTBI should be cautiously monitored. The second patient started a multidrug anti-TB regimen, but the diagnosis of active TB was finally infirmed. In contrast, the third patient was diagnosed with LTBI and was treated successfully with biologic therapy for more than 2 years, despite a short course of a chemoprophylactic regimen with isoniazid.

TNF-alpha is a pro-inflammatory cytokine that stimulates the acute phase reaction. It has a broad spectrum of biologic effects: it stimulates inflammatory cytokines (interleukin [IL]-1beta, IL-6, IL-8, granulocyte-macrophage colonystimulating factor [GM-CSF]) and chemokines (monocyte chemotactic protein-1 [MCP-1], Macrophage inflammatory protein [MIP]1alpha, MIP-2, RANTES [regulated and normal $T$ cell expressed and secreted]) [12], activates endothelial adhesion molecules (vascular cell adhesion molecule 1 [VCAM-1], intercellular Adhesion Molecule 1 [ICAM-1], E-selectin), induces apoptosis, and inhibits tumorigenesis and viral replication. TNF-alpha is important in the protection against $M$. tuberculosis through its role in granuloma formation. It recruits macrophages and lymphocytes, and is required for the maintenance of the granulomatous structure $[13,14]$.

The cytokines released by $\mathrm{T}$ cells play a critical role in $\mathrm{TB}$ pathogenesis through macrophage activation and granuloma formation. T-helper 1 (Th1) lymphocytes release interferon-gamma (IFN- $\gamma$ ) and TNFalpha. These cytokines are involved in the transformation of macrophages into specialized histiocytic cells with bactericidal and bacteriostatic functions. Activated 
macrophages, under T-lymphocyte influence, organize and form the tuberculoid granulomas. In contrast, TNF-blockade is associated with granuloma lysis $[9,15]$.

Many randomized, controlled studies have evaluated the safety of etanercept, infliximab, and adalimumab $[16,17]$, the majority of which have been conducted in patients with rheumatologic conditions or Crohn's disease. However, according to the Food and Drug Administration (FDA) Adverse Event Reporting System (AERS), only a single case of TB occurred during initial clinical trials of infliximab [18] and none of the patients treated with etanercept and adalimumab developed TB during the initial studies [9]. Despite these results, TB has been continuously reported in association with biologic therapy [19-22] .

Data from the British Society for Rheumatology Biologics Register (BSRBR), analyzing 10,712 patients with rheumatoid arthritis treated with anti-TNF agents, reported 39 cases of active TB. The risk for TB was as follows: 144 events/100,000 patient-years for adalimumab; 136/100,000 patient-years for infliximab; and 39/100,000 patient-years for etanercept, confirming that infliximab and adalimumab are associated with a three- to fourfold higher rate of $\mathrm{TB}$ compared with etanercept. The median time to TB diagnosis was 13.4 months for patients exposed to etanercept, 5.5 months for infliximab, and 18.5 months for patients exposed to adalimumab [20]. Other publications have indicated a lower risk of TB in patients treated with etanercept compared with infliximab or adalimumab [17, 22-27].

The safety data from patients with rheumatoid arthritis can only partially be generalized to patients with psoriasis vulgaris, as psoriasis is typically treated with monotherapy whereas rheumatoid arthritis is commonly based on treatment regimens consisting of systemic immunosuppressants and biologics, which can increase the risk of infection [28].

The present authors searched the MEDLINE database for randomized, placebo-controlled studies of the three currently used anti-TNF agents (infliximab, etanercept, and adalimumab) published between 2003 and 2012. Study participants were adult patients with moderate-to-severe psoriasis treated with anti-TNF agents for at least 12 weeks. Based on these criteria, 13 clinical trials [29-41] were identified that collectively included 3,657 adult patients with moderate-to-severe psoriasis who were treated with adalimumab, etanercept, or infliximab (Table 2). The total number of patients receiving the placebo was 1,709 . The treatment duration ranged from 12 to 52 weeks. All trials compared anti-TNF agents with the placebo: three trials with adalimumab, six trials with etanercept, and four trials with infliximab. Three studies [29, 40,41] reported active TB as an adverse event occurring during anti-TNF therapy: one patient was treated with adalimumab and five patients received infliximab. Active TB was not reported in the placebo group.

Although clinical trials have demonstrated significant efficacy and a low number of TB cases in patients with psoriasis, questions remain about the long-term use of these agents. There are several limitations that make it difficult to assess the potential for anti-TNF therapy to promote TB infection. For example, the median time to $\mathrm{TB}$ diagnosis has been reported to range from 5.5 to 18.5 months [20], and these randomized, controlled studies extend to a limited period of time (3-13 months). From another point of view, the study of Yang et al. [41] highlights that $\mathrm{TB}$ is a major problem in endemic areas. 


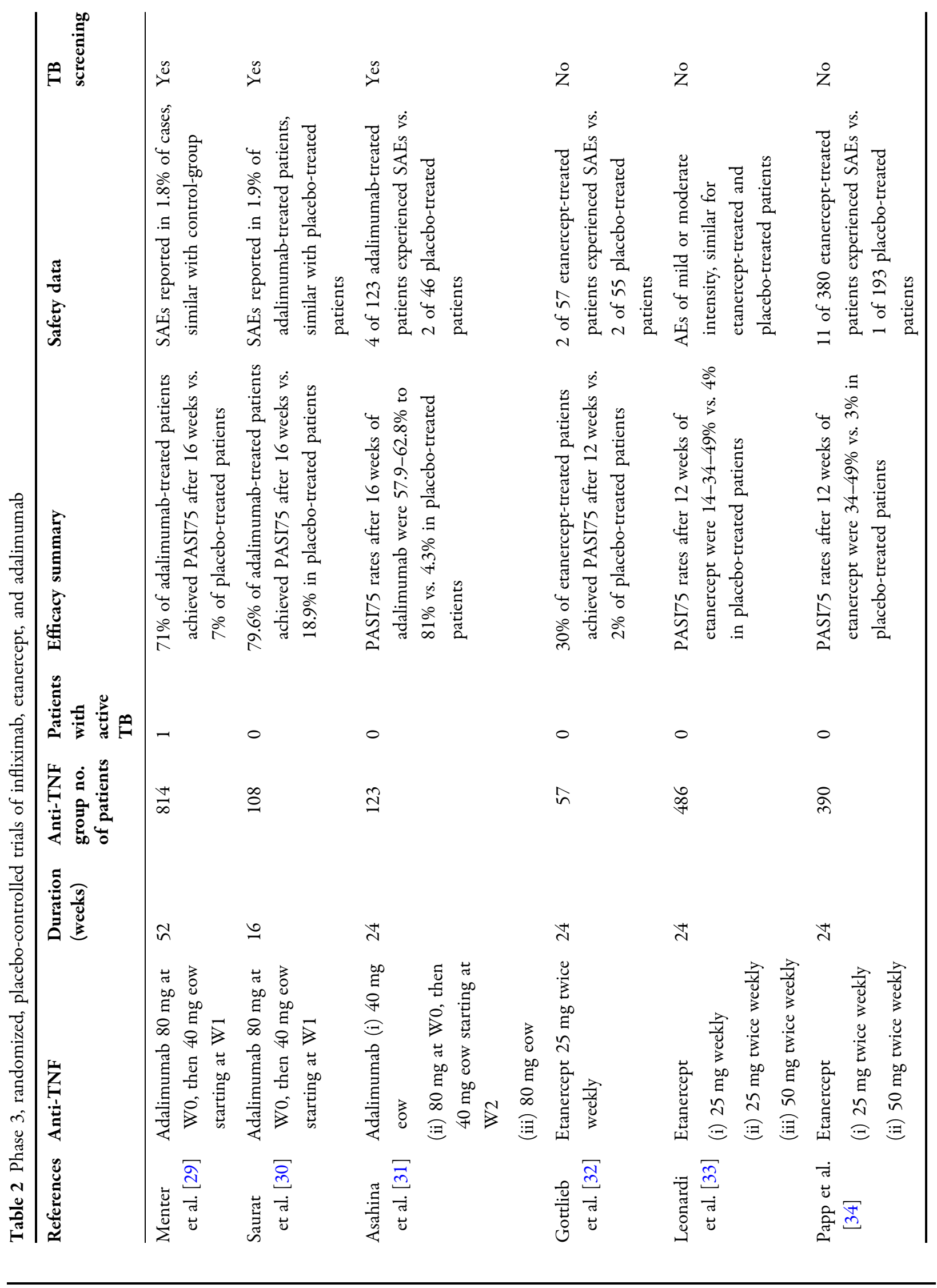




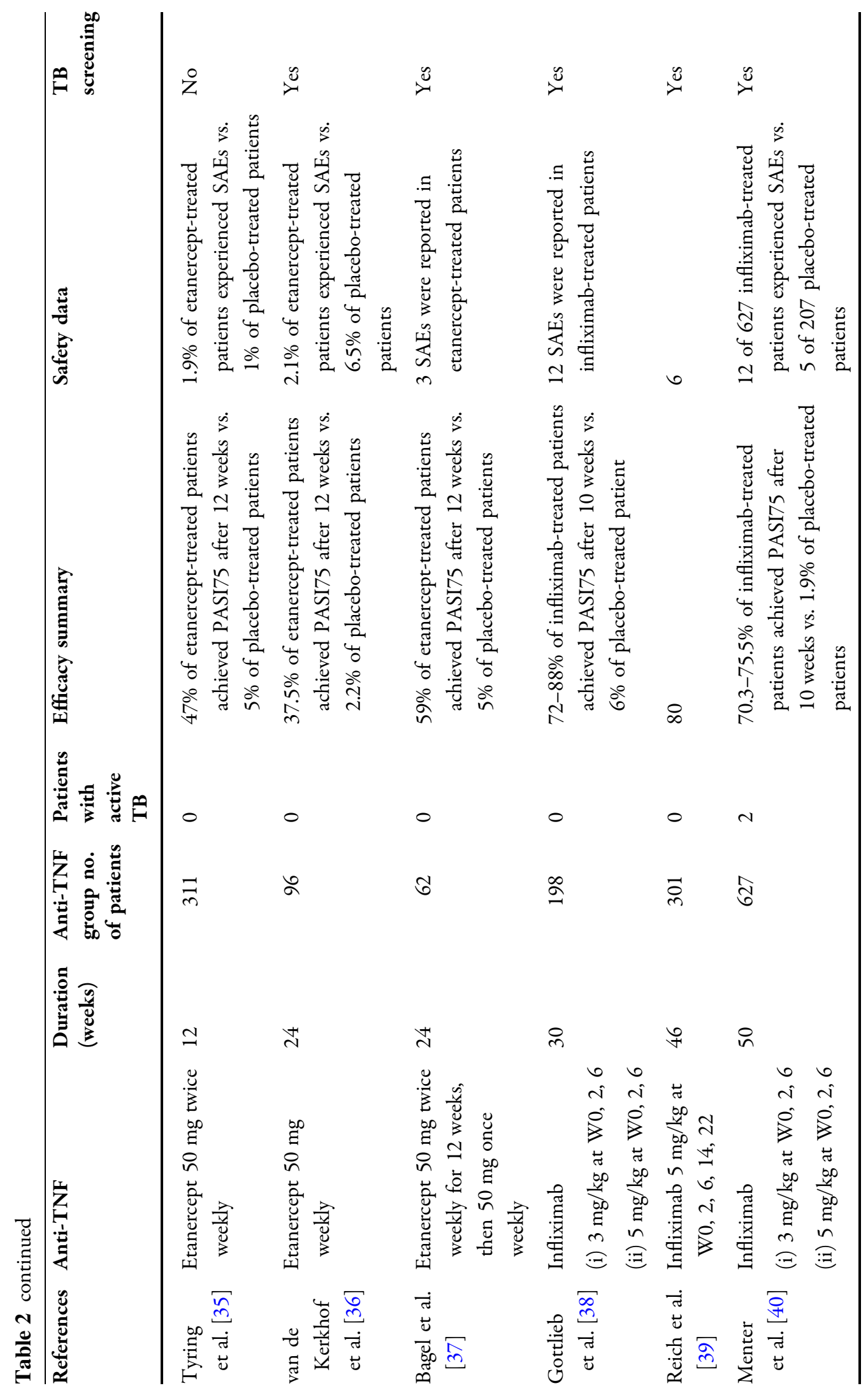




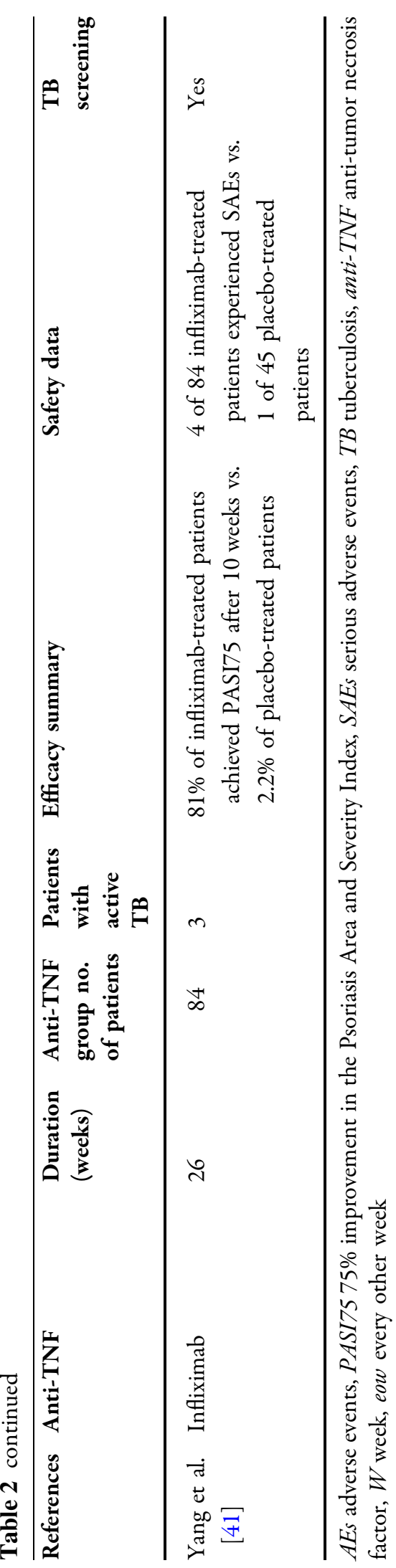

Furthermore, clinical practice continues to provide details concerning the increasing numbers of patients with active $\mathrm{TB}$, despite the screening methods for detecting LTBI [42-47].

TB often presents as extrapulmonary or disseminated disease in such patients and has been reported with the use of all of the anti-TNF agents $[15,18,21,48-51]$. This form of presentation is explained by the underlying mechanism: the immunosuppression induced by anti-TNF therapy leads to reactivation of secondary foci and dissemination of M. tuberculosis [52]. The monoclonal antibodies form stable complexes with all forms of TNFalpha, including TNF on the surface of macrophages and $\mathrm{T}$ cells, which induces $\mathrm{T}$ cell and macrophage apoptosis $[53,54]$. In addition, biologic therapy inhibits the Th1 cell response, as well as the production of IFN, a cytokine with major roles in the immune defense against $M$. tuberculosis $[55,56]$. Thus, these actions disturb granuloma integrity and increase the risk of secondary foci reactivation [52].

Active TB associated with biologic treatment is believed to be the result of LTBI reactivation in most cases. LTBI is defined as a complex clinical condition in which an infection with M. tuberculosis persists in a subclinical status with minimal replication. The bacilli are unable to cause clinical manifestations and cannot be identified in culture [57]. Identification of latent infection and chemoprophylaxis with isoniazid seems to decrease the risk of developing active disease [58]. Thus, an important prophylactic measure is the treatment of LTBI [59]. This approach reduces the reactivation risk by over $80 \%[60,61]$. However, de novo TB has also been reported $[62,63]$. A short time to the onset of TB after the start of biologic treatment suggests LTBI reactivation as the new infections seem to occur at random during 
anti-TNF treatment. De novo TB is not influenced by anti-LTBI treatments. In these cases, new approaches are required, such as primary prevention [64].

Although current guidelines recommend screening prior to anti-TNF therapy, there are no standard indications and there is a lack of consensus on interpreting TST in patients with psoriasis. The consensus guidelines from the National Psoriasis Foundation, USA, state that an induration $>5 \mathrm{~mm}$ is classified as positive in patients with immunosuppression, including patients who are receiving TNF antagonists [7]. The main disadvantage is that they do not provide specific guidelines on interpreting TST for patients about to start anti-TNF therapy [8]. Some authors consider that skin indurations of $5 \mathrm{~mm}$ or greater should be interpreted as a positive result for LTBI in any patient considered for TNF blockade [65]. This cut-off value is accepted by most guidelines, including the national guidelines, but it may overestimate LTBI in psoriatic patients, leading to unnecessary treatments. The present authors previously reported that patients with moderate-to-severe psoriasis had positive TST reactions more frequently $(70.5 \%)$ than nondermatologic subjects (51\%) [66]. Although the TST still represents a useful method, it is difficult to perform and read in psoriatic patients with extensive lesions, because these patients rarely present clinically unaffected skin for testing. Moreover, important immunologic mechanisms take place in even apparently healthy skin of psoriatic patients; the proinflammatory state can lead to an overreaction to antigenic triggers [67]. Another factor that may lead to false-positive results is the Koebner phenomenon (development of psoriatic lesions at the site of trauma), reported after intradermal injection of purified protein derivative (PPD) in psoriatic patients [68]. In contrast, psoriatic patients with negative TST results and positive QFT-G results have been reported [69-71]. The reversion of a positive TST result to a negative result may also occur [72]. Thus, to minimize the risk of false-negative results, some authors propose a booster dose 7-10 days after an initially negative TST [73]. Tubach et al. [3] reported 69 cases of TB in patients treated with anti-TNF agents, two-thirds of which occurred in patients with negative TST results at screening. However, the authors suggested that both reactivation of LTBI during the first year of treatment and new infections occurring during follow-up were responsible for the high incidence of TB reported in their study.

IGRAs are alternative tools for TB diagnosis. The principle of these methods is based on the detection of IFN- $\gamma$ produced by the effectors memory $\mathrm{T}$ cells upon in vitro stimulation with the TB-specific antigens, early secretory antigen (ESAT) 6 and culture filtrate protein (CFP) 10. IFN can be measured using either ELISpot-based assay, represented by $\mathrm{T}_{\text {-SPOT }}{ }^{\circledR}$.TB (Immunotech, Abingdon, UK), or an enzymelinked immunosorbent assay (ELISA), represented by QFT-G and QFT-in-tube (QFTIT; Cellestis, Victoria, Australia) [74]. Although QFT-G demonstrates high specificity for LTBI (96-99\%), its sensitivity is still questionable (70-78\%) [75]. In one study, LTBI treatment was avoided in $20 \%$ of patients with positive TST results but negative IGRA results [76].

The use of both methods in parallel can enhance both sensitivity and specificity. Furthermore, routine periodic retesting during therapy could allow for the detection of possible conversions. However, serial TST testing is not strictly recommended due to the boosting effect [60]. There is also evidence that the TST can boost subsequent IGRA results. The effect is evident after the first 3 days post-TST testing and potentially wanes after a few months [77]. Furthermore, the 
use of IGRAs during immunosuppressive treatment (including biologic therapy) is controversial, because the immunosuppression might decrease the production of IFN and interfere with the results [74].

Another inconvenience for both TST and IGRAs is the lack of discrimination between latent and active TB [60]. Positive TST/IGRAs tests at baseline often remain positive despite a successful anti-TB treatment. In these cases careful monitoring for clinical signs and symptoms of active TB is recommended [78].

According to the Tuberculosis Network European Trials Group (TBNET) consensus, the chemoprophylactic regimens recommended for LTBI include 6 or 9 months with isoniazid, 3 months of rifampicin plus isoniazid, or 4 months of rifampicin [79]. Another regimen used in the USA includes rifampicin and pyrazinamide for 2 months, although this regimen has been associated with a high number of side effects [80].

The diagnostic tools for active TB infection include clinical assessment, cultures for $M$. tuberculosis, staining for acid-fast bacilli, chest $\mathrm{X}$-rays, and nucleic acid amplification assays [9]. Although culture is considered the reference standard, in clinical practice the diagnosis and treatment of TB are usually based on the presence of abnormal radiologic findings or clinical suspicion [20].

The recommendations for resuming biologic therapy in active TB patients are controversial. According to the American College of Rheumatology (ACR), anti-TNF therapy can be initiated or resumed after 1 month of chemoprophylaxis for LTBI and after completion of therapy for active disease [78]. The British Society for Rheumatology (BSR) accepts the continuation of biologic therapy during TB treatment if clinically indicated [81]. Hernandez et al. [82] reported 27 patients with active TB that resumed anti-TNF therapy before completing tuberculostatic treatment, without relapse during a 4-year follow-up.

In conclusion, anti-TNF agents are an established option for the treatment of psoriasis, but the safety profile should be carefully monitored. Even otherwise healthy patients with no predisposing factors for TB should be cautiously managed during biologic therapy. It is mandatory for the dermatologists who prescribe anti-TNF agents to carefully evaluate the patients to exclude concomitant $\mathrm{TB}$ and non-TB infections. Continuous vigilance, long-term follow-up, and systematic reporting of any suspected association between active TB and biologic therapy will improve the prevention and management of this complication.

\section{ACKNOWLEDGMENTS}

This work was not supported financially or otherwise. Dr. Chiticariu is the guarantor for this article, and takes responsibility for the integrity of the work as a whole.

Conflict of interest. Dr. Solovan has no conflict of interest to disclose. Dr. Chiticariu has no conflict of interest to disclose.

Open Access. This article is distributed under the terms of the Creative Commons Attribution Noncommercial License which permits any noncommercial use, distribution, and reproduction in any medium, provided the original author(s) and the source are credited.

\section{REFERENCES}

1. Parisi R, Symmons DP, Griffiths CE, et al. Global epidemiology of psoriasis: a systematic review of incidence and prevalence. J Invest Dermatol. 2013;133:377-85. 
2. Menter A, Gottlieb A, Feldman SR, et al. Guidelines of care for the management of psoriasis and psoriatic arthritis: section 1. Overview of psoriasis and guidelines of care for the treatment of psoriasis with biologics. J Am Acad Dermatol. 2008;58: 826-50.

3. Tubach F, Salmon-Céron D, Ravaud P, et al. Risk of tuberculosis is higher with anti-tumor necrosis factor monoclonal antibody therapy than with soluble tumor necrosis factor receptor therapy: the three-year prospective French Research Axed on Tolerance of Biotherapies registry. Arthritis Rheum. 2009;60:1884-99.

4. Global tuberculosis report 2012, World Health Organization, 2012. http://www.who.int/tb/ publications/global_report/en/. Accessed Jan 28, 2013.

5. Sánchez-Moya AI, Dauden E. Incidence of tuberculosis infection in psoriatic patients on antiTNF therapy: report of a case series with 144 patients. J Eur Acad Dermatol Venereol. 2011;25:730-3.

6. Denkinger CM, Dheda K, Pai M. Guidelines on interferon- $\gamma$ release assays for tuberculosis infection: concordance, discordance or confusion? Clin Microbiol Infect. 2011;17:806-14.

7. Doherty SD, Van Voorhees A, Lebwohl MG, et al. National Psoriasis Foundation consensus statement on screening for latent tuberculosis infection in patients with psoriasis treated with systemic and biologic agents. J Am Acad Dermatol. 2008;59: 209-17.

8. Brown AJ, Lesher JL Jr. Anti-tumor necrosis factor therapy and interpreting tuberculin skin tests. J Am Acad Dermatol. 2009;60:e21-2.

9. Hernandez C, Cetner AS, Jordan JE, et al. Tuberculosis in the age of biologic therapy. J Am Acad Dermatol. 2008;59:363-80.

10. Pathirana D, Ormerod AD, Saiag P, et al. European S3-guidelines on the systemic treatment of psoriasis vulgaris. J Eur Acad Dermatol Venereol. 2009; 23(Suppl. 2):1-70.

11. Kardos M, Kimball AB. Time for a change? Updated guidelines using interferon gamma release assays for detection of latent tuberculosis infection in the office setting. J Am Acad Dermatol. 2012;66: 148-52.

12. Roach DR, Bean AG, Demangel C, et al. TNF regulates chemokine induction essential for cell recruitment, granuloma formation, and clearance of mycobacterial infection. J Immunol. 2002;168: 4620-7.
13. Raychaudhuri SP, Nguyen CT, Raychaudhuri SK, et al. Incidence and nature of infectious disease in patients treated with anti-TNF agents. Autoimmun Rev. 2009;9:67-81.

14. Hartmann P, Plum G. Immunological defense mechanisms in tuberculosis and MAC-infection. Diagn Microbio Infect Dis. 1999;34:147-52.

15. Keane J, Gershon SK, Braun MM. Tuberculosis and treatment with infliximab. N Engl J Med. 2002;346: 623-6.

16. Alonso-Ruiz A, Pijoan JI, Ansuategui E, et al. Tumor necrosis factor alpha drugs in rheumatoid arthritis: systematic review and metaanalysis of efficacy and safety. BMC Musculoskelet Disord. 2008;9:52.

17. Peyrin-Biroulet L. Anti-TNF therapy in inflammatory bowel diseases: a huge review. Minerva Gastroenterol Dietol. 2010;56:233-43.

18. Mohan AK, Coté TR, Block JA, et al. Tuberculosis following the use of etanercept, a tumor necrosis factor inhibitor. Clin Infect Dis. 2004;39:295-9.

19. Schiff $M H$, Burmester GR, Kent JD, et al. Safety analyses of adalimumab (HUMIRA) in global clinical trials and US post-marketing surveillance of patients with rheumatoid arthritis. Ann Rheum Dis. 2006;65:889-94.

20. Dixon WG, Hyrich KL, Watson KD, et al. Drugspecific risk of tuberculosis in patients with rheumatoid arthritis treated with anti-TNF therapy: results from the British Society for Rheumatology Biologics Register (BSRBR). Ann Rheum Dis. 2010;69:522-8.

21. Wallis RS, Broder M, Wong J, et al. Granulomatous infections due to tumor necrosis factor blockade: correction. Clin Infect Dis. 2004;39:1254-5.

22. Gomez-Reino JJ, Carmona L, Valverde VR, et al. Treatment of rheumatoid arthritis with tumor necrosis factor inhibitors may predispose to significant increase in tuberculosis risk: a multicenter active-surveillance report. Arthritis Rheum. 2003;48:2122-7.

23. Fonseca JE, Canhao H, Silva C, et al. Tuberculosis in rheumatic patients treated with tumour necrosis factor alpha antagonists: the Portuguese experience. Acta Reumatol Port. 2006;31:247-53.

24. Brassard P, Kezouh A, Suissa S. Antirheumatic drugs and the risk of tuberculosis. Clin Infect Dis. 2006;43:717-22.

25. Tubach F, Salmon-Céron D, Ravaud P, et al. The RATIO observatory: French registry of opportunistic infections, severe bacterial infections, and 
lymphomas complicating anti-TNFalpha therapy. Jt Bone Spine. 2005;72:456-60.

26. Ehlers S. Tumor necrosis factor and its blockade in granulomatous infections: differential modes of action of infliximab and etanercept? Clin Infect Dis. 2005;41(Suppl. 3):S199-203.

27. Wallis RS, Kyambadde P, Johnson JL, et al. A study of the safety, immunology, virology, and microbiology of adjunctive etanercept in HIV-1associated tuberculosis. AIDS. 2004;18:257-64.

28. Dommasch ED, Abuabara K, Shin DB, et al. The risk of infection and malignancy with tumor necrosis factor antagonists in adults with psoriatic disease: a systematic review and meta-analysis of randomized controlled trials. J Am Acad Dermatol. 2011;64: 1035-50.

29. Menter A, Tyring SK, Gordon K, et al. Adalimumab therapy for moderate to severe psoriasis: a randomized, controlled phase III trial. J Am Acad Dermatol. 2008;58:106-15.

30. Saurat JH, Stingl G, Dubertret L, et al. Efficacy and safety results from the randomized controlled comparative study of adalimumab vs. methotrexate vs. placebo in patients with psoriasis (CHAMPION). Br J Dermatol. 2008;158:558-66.

31. Asahina A, Nakagawa $H$, Etoh $T$, Ohtsuki M, Adalimumab MO4-688 Study Group. Adalimumab in Japanese patients with moderate to severe chronic plaque psoriasis: efficacy and safety results from a phase II/III randomized controlled study. J Dermatol. 2010;37:299-310.

32. Gottlieb AB, Matheson RT, Lowe N, et al. A randomized trial of etanercept as monotherapy for psoriasis. Arch Dermatol. 2003;139:1627-32.

33. Leonardi CL, Powers JL, Matheson RT, et al. Etanercept as monotherapy in patients with psoriasis. N Engl J Med. 2003;349:2014-22.

34. Papp KA, Tyring S, Lahfa M, et al. A global phase III randomized controlled trial of etanercept in psoriasis: safety, efficacy, and effect of dose reduction. Br J Dermatol. 2005;152:1304-12.

35. Tyring S, Gottlieb A, Papp K, et al. Etanercept and clinical outcomes, fatigue, and depression in psoriasis: double-blind placebo-controlled randomized phase III trial. Lancet. 2006;367:29-35.

36. van de Kerkhof PC, Segaert S, Lahfa M, et al. Once weekly administration of etanercept $50 \mathrm{mg}$ is efficacious and well tolerated in patients with moderate-to-severe plaque psoriasis: a randomized controlled trial with open-label extension. $\mathrm{Br} \mathrm{J}$ Dermatol. 2008;159:1177-85.
37. Bagel J, Lynde C, Tyring S, et al. Moderate to severe plaque psoriasis with scalp involvement: a randomized, double-blind, placebo-controlled study of etanercept. J Am Acad Dermatol. 2012;67: 86-92.

38. Gottlieb AB, Evans R, Li $\mathrm{S}$, et al. Infliximab induction therapy for patients with severe plaquetype psoriasis: a randomized, double-blind, placebo-controlled trial. J Am Acad Dermatol. 2004;51:534-42.

39. Reich K, Nestle FO, Papp $K$, et al. Infliximab induction and maintenance therapy for moderateto-severe psoriasis: a phase III, multicentre, doubleblind trial. Lancet. 2005;366:1367-74.

40. Menter A, Feldman SR, Weinstein GD, et al. A randomized comparison of continuous vs. intermittent infliximab maintenance regimens over 1 year in the treatment of moderate-to-severe plaque psoriasis. J Am Acad Dermatol. 2007;56: 31.e1-15.

41. Yang $\mathrm{HZ}$, Wang $\mathrm{K}$, Jin $\mathrm{HZ}$, et al. Infliximab monotherapy for Chinese patients with moderate to severe plaque psoriasis: a randomized, doubleblind, placebo-controlled multicenter trial. Chin Med J (Engl). 2012;125:1845-51.

42. Shaikha SA, Mansour K, Riad H. Reactivation of tuberculosis in three cases of psoriasis after initiation of anti-TNF therapy. Case Rep Dermatol. 2012;4:41-6.

43. Gori A, Fabroni C, Prignano F, et al. Unusual presentation of tuberculosis in an infliximabtreated patient-which is the correct TB screening before starting a biologic? Dermatol Ther. 2010;23(Suppl. 1):S1-3.

44. Fortaleza GT, Brito Mde F, Santos JB, et al. Splenic tuberculosis during psoriasis treatment with infliximab. An Bras Dermatol. 2009;84: 420-4.

45. Letada PR, Hitchcock E, Steele SL, et al. Transient improvement in chronic psoriasis after treatment of TNF- $\alpha$ blocker induced disseminated M. tuberculosis infection. J Drugs Dermatol. 2012;11:119-20.

46. Perlmutter A, Mittal A, Menter A. Tuberculosis and tumour necrosis factor-alpha inhibitor therapy: a report of three cases in patients with psoriasis. Comprehensive screening and therapeutic guidelines for clinicians. Br J Dermatol. 2009;160: $8-15$.

47. Huo R, Romanelli P. Etanercept therapy for psoriasis in a patient with active pulmonary tuberculosis. Am J Clin Dermatol. 2010;11(Suppl. 1):39-40. 
48. Moustou AE, Matekovits A, Dessinioti C, et al. Cutaneous side effects of anti-tumor necrosis factor biologic therapy: a clinical review. J Am Acad Dermatol. 2009;61:486-504.

49. Burmester GR, Mease P, Dijkmans BA, et al. Adalimumab safety and mortality rates from global clinical trials of six immune-mediated inflammatory diseases. Ann Rheum Dis. 2009;68:1863-9.

50. Furst DE, Keystone EC, Fleischmann R, et al. Updated consensus statement on biological agents for the treatment of rheumatic diseases, 2009. Ann Rheum Dis. 2010;69:i2-29.

51. Emery P, Fleischmann RM, Moreland LW, et al. Golimumab, a human anti-tumor necrosis factor alpha monoclonal antibody, injected subcutaneously every four weeks in methotrexatenaive patients with active rheumatoid arthritis: twenty-four-week results of a phase III, multicenter, randomized, double-blind, placebocontrolled study of golimumab before methotrexate as first-line therapy for early-onset rheumatoid arthritis. Arthritis Rheum. 2009;60: 2272-83.

52. Lukacs NW, Chensue SW, Strieter RM, Warmington $\mathrm{K}$, Kunkel SL. Inflammatory granuloma formation is mediated by TNF-alpha-inducible intercellular adhesion molecule-1. J Immunol. 1994;152: 5883-9.

53. Mitoma $\mathrm{H}$, Horiuchi $\mathrm{T}$, Hatta $\mathrm{N}$, et al. Infliximab induces potent anti-inflammatory responses by outside-to-inside signals through transmembrane TNF-alpha. Gastroenterology. 2005;128:376-92.

54. van den Brande J, Hommes DW, Peppelenbosch MP. Infliximab induced T lymphocyte apoptosis in Crohn's disease. J Rheumatol Suppl. 2005;74:26-30.

55. Saliu OY, Sofer C, Stein DS, et al. Tumor necrosisfactor blockers: differential effects on mycobacterial immunity. J Infect Dis. 2006;194:486-92.

56. Wallis RS. Reactivation of latent tuberculosis by TNF blockade: the role of interferon gamma. J Investig Dermatol Symp Proc. 2007;12:16-21.

57. Mack U, Migliori GB, Sester $M$, et al. Latent tuberculosis infection or lasting immune responses to $M$. tuberculosis? A TBNET consensus statement. Eur Respir J. 2009;33:956-73.

58. Keane J. TNF-blocking agents and tuberculosis: new drugs illuminate an old topic. Rheumatology (Oxford). 2005;44:714-20.

59. Balato N, Di Costanzo L, Ayala F, Blato A, Sanduzzi A, Bocchino H. Psoriatic disease and tuberculosis nowadays. Clin Dev Immunol. 2012;2012:747204.
60. Furst DE, Breedveld FC, Kalden JR, et al. Updated consensus statement on biological agents, specifically tumour necrosis factor \{alpha\} (TNF\{alpha\}) blocking agents and interleukin-1 receptor antagonist (IL-1ra), for the treatment of rheumatic diseases, 2005. Ann Rheum Dis. 2005;64(Suppl. 4):iv2-14.

61. Carmona L, Gomez-Reino JJ, Rodriguez-Valverde V, et al. Effectiveness of recommendations to prevent reactivation of latent tuberculosis infection in patients treated with tumor necrosis factor antagonists. Arthritis Rheum. 2005;52:1766-72.

62. Arend SM, Leyten EM, Franken WP, et al. A patient with de novo tuberculosis during anti-tumor necrosis factor-alpha therapy illustrating diagnostic pitfalls and paradoxical response to treatment. Clin Infect Dis. 2007;45:1470-5.

63. Abud-Mendoza C, Martínez-Martínez MU, DE Jesús Macías-Mendoza J, et al. Should tuberculin skin test be positive to give latent tuberculosis treatment before tumor necrosis factor-alpha inhibitors in selected patients in developing countries? J Rheumatol. 2010;37:672-3.

64. Wallis RS. Mathematical modeling of the cause of tuberculosis during tumor necrosis factor blockade. Arthritis Rheum. 2008;58:947-52.

65. Winthrop KL. Risk and prevention of tuberculosis and other serious opportunistic infections associated with the inhibition of tumor necrosis factor. Nat Clin Pract Rheumatol. 2006;2:602-10.

66. Solovan C, Chiticariu E, Timofte A, Stoia-Djeska I. Tuberculosis infection versus anti-tumor necrosis factor therapy: screening challenges in psoriatic patients. J Drug Assess. 2012;1:65-7.

67. Tsiouri G, Gaitanis G, Kiorpelidou D, et al. Tuberculin skin test overestimates tuberculosis hypersensitivity in adult patients with psoriasis. Dermatology. 2009;219:119-25.

68. Dogan B, Harmanyeri Y. Intradermal antigen tests and the Koebner phenomenon in psoriasis. Int $\mathrm{J}$ Dermatol. 1997;36:263-5.

69. Haddican MM, Koo JY. Is tuberculin skin testing reliable during anti-tumor necrosis factor-alfa therapy? A case report and review of the literature. J Am Acad Dermatol. 2011;65:195-7.

70. Bartalesi F, Vicidomini S, Goletti $\mathrm{D}$, et al. QuantiFeron-TB-Gold and the TST are both useful for latent tuberculosis infection screening in autoimmune diseases. Eur Respir J. 2009;33:586-93.

71. Chen DY, Shen GH, Hsieh TY, et al. Effectiveness of the combination of a whole-blood interferon- 
gamma assay and the tuberculin skin test in detecting latent tuberculosis infection in rheumatoid arthritis patients receiving adalimumab therapy. Arthritis Rheum. 2008;59:800-6.

72. Menzies D. Interpretation of repeated tuberculin tests: boosting, conversion, and reversion. Am J Respir Crit Care Med. 1999;159:15-21.

73. Gomez-Reino JJ, Carmona L, Angel Descalzo M, Biobadaser Group. Risk of tuberculosis in patients treated with tumor necrosis factor antagonists due to incomplete prevention of reactivation of latent infection. Arthritis Rheum. 2007;57:756-61.

74. Lalvani A, Millington KA. Screening for tuberculosis infection prior to initiation of anti-TNF therapy. Autoimmun Rev. 2008;8:147-52.

75. Pai M, Zwerling A, Menzies D. Systematic review: T-cell-based assays for the diagnosis of latent tuberculosis infection: an update. Ann Intern Med. 2008;149:177-84.

76. Chiang YZ, Panting $\mathrm{K}$, Dever $\mathrm{B}$, et al. Clinical applicability of T-cell interferon- $\alpha$ release assay for tumour necrosis factor- $\alpha$ inhibitor therapy in severe psoriasis. Clin Exp Dermatol. 2011;36:39-41.

77. van Zyl-Smit RN, Zwerling A, Dheda $\mathrm{K}$, et al. Within-subject variability of interferon-g assay results for tuberculosis and boosting effect of tuberculin skin testing: a systematic review. PLoS One. 2009;4:e8517.

78. Singh JA, Furst DE, Bharat A, et al. 2012 update of the 2008 American College of Rheumatology recommendations for the use of disease-modifying antirheumatic drugs and biologic agents in the treatment of rheumatoid arthritis. Arthritis Care Res (Hoboken). 2012;64:625-39.

79. Solovic I, Sester M, Gomez-Reino JJ, et al. The risk of tuberculosis related to tumour necrosis factor antagonist therapies: a TBNET consensus statement. Eur Respir J. 2010;36:1185-206.

80. Stout JE, Engemann JJ, Cheng AC, et al. Safety of 2 months of rifampin and pyrazinamide for treatment of latent tuberculosis. Am J Respir Crit Care Med. 2003;167:824-7.

81. Ding T, Ledingham J, Luqmani R, et al. BSR and BHPR rheumatoid arthritis guidelines on safety of anti-TNF therapies. Rheumatology (Oxford). 2010;49:2217-9.

82. Hernandez MV, Descalzo MA, Canete JD, et al. When can biological therapy be resumed in patients with rheumatic conditions who develop tuberculosis infection during tumour necrosis factors antagonists therapy? Study based on the Biobadaser Data Registry. Arthritis Rheum. 2012;64:S701-2. 